\title{
Tratamento cirúrgico da endomiocardiofibrose: substituição valvar versus técnicas conservadoras
}

\author{
José Carlos R. IGLÉZIAS *, Luís Alberto DALLAN *, Antônio Carlos PEREIRA-BARRETO *, Charles \\ MADY *, Protásio Lemos da LUZ *, Noedir A. G. STOLF *, Sérgio Almeida de OLIVEIRA *, Fúlvio \\ PILEGGI *, Adib D. JATENE *
}

\section{RBCCV 44205-189}

IGLÉZIAS, J. C. R.; DALLAN, L. A.; PEREIRA-BARRETO, A. C.; MADY, C.; LUZ, P. L.; STOLF, N. A. G.; OLIVEIRA, S. A.; PILEGGI, F.; JATENE, A. D. - Tratamento cirúrgico da endomiocardiofibrose: substituiçāo valvar versus técnicas conservadoras. Rev. Bras. Cir. Cardiovasc., 7(4):269-274, 1992.

RESUMO: No Instituto do Coraçāo do HC da FM-USP, no período compreendido entre janeiro de 1978 e junho de 1990, 66 pacientes portadores de endomiocardiofibrose (EMF) foram tratados cirurgicamente com a finalidade de comparar as técnicas operatórias empregadas. Foi elaborado um estudo retrospectivo envolvendo dois subgrupos: $1^{\circ}$ ) pacientes submetidos a endocardiectomia e substituiçāo valvar e $2^{\circ}$ ) pacientes submetidos a endocardiectomia e plastia valvar. No roteiro foram incluídos parâmetros clínicos, radiológicos, hemodinâmicos, cirúrgicos e de pós-operatório. Tratou-se de um estudo retrospectivo e as análises estatísticas foram realizadas por meio do teste do $\mathrm{X}^{2}$ de Pearson e do teste de Loong-Rank. Embora a análise comparativa não revele, no momento do estudo, diferenças significantes estatisticamente entre os subgrupos, a observaçăo das curvas atuariais de sobrevida permitem identificar uma forte tendência para melhor (menor mortalidade hospitalar e maior sobrevida tardia) com a utilizaçäo das técnicas conservadoras.

DESCRITORES: endomiocardiofibrose, cirurgia; valvas cardiacas, cirurgia.

\section{INTRODUÇĀO}

A endocardite fibrosa constritiva descrita inicialmente por Loeffler em 1936, consiste na fibrose endocárdica de vários milímetros de espessamento a qual é potencialmente constrictiva e limitada às cavidades ventriculares.

A evolução clínica é caracterizada pela evolução em direção à insuficiência cardíaca a qual pode ser predominantemente direita ou esquerda e conduzir rapidamente ao óbito.

Os recursos tecnológicos modernos, tais como a cateterização cardíaca e a angiocardiografia, definiram as características hemodinâmicas da molés- tia como sendo causada pela disfunçāo ventricular localizada no lado direito, esquerdo ou bilateral, assim como a assitolia.

A etiologia permanece obscura e as alteraçōes patológicas nāo são específicas.

A evolução invariável para o óbito estímulou consideraçōes de outras possibilidades para o tratamento cirúrgico.

A endocardiectomia foi inicialmente realizada por DUBOST et alii ${ }^{3} \mathrm{e}$ BARRETO et alii' $\mathrm{e}$ com melhora clínica para os pacientes. Desde então outros pacientes foram considerados tanto para endocardiectomia direita como esquerda ou combi-

Trabalho realizado no Instituto do Coração do Hospital das Clínicas da Faculdade de Medicina da Universidade de São Paulo. São Paulo, SP. Brasil. Apresentado ao $19^{\circ}$ Congresso Nacional de Cirurgia Cardiaca., Sảo Paulo. SP. 7 a 9 de maio. 1992.

* Do Instituto do Coração do Hospital das Clínicas da Faculdade de Medicina da Universidade de São Paulo.

Endereço para separatas: José Carlos Iglézias. Av. Dr. Enéas Carvalho de Aguiar, 44. Divisảo Cirúrgica. 05403 São Paulo, SP, Brasil. 
IGLÉZIAS, J. C. R.; DALLAN, L. A.; PEREIRA-BARRETO, A. C.; MADY, C.; LUZ, P. L.; STOLF, N. A. G.; OLIVEIRA, S. A.; PILEGGI, F.; JATENE, A. D. - Tratamento cirúrgico da endomiocárdiofibrose: substituiçăo valvar versus técnicas conservadoras. Rev. Bras. Cir. Cardiovasc., 7(4):269-274, 1992.

nada e năo houve dúvida de que o melhor conhecimento dos aspectos clínicos da moléstia também colaboraram para o aumento da casuística.

O desenvolvimento da endomiocardiofibrose é semelhante em todos os casos. Ela envolve as câmaras de enchimento de um ou ambos os ventrículos, inclusive os músculos papilares, cordas tendíneas das valvas mitral e tricúspide, que estão envolvidas no processo na maioria dos casos.

O espessamento do processo fibroso varia de $4 \mathrm{~mm}$ a $10 \mathrm{~mm}$. O miocárdio subjacente apresenta lesōes fibrosas que sāo localizadas na camada subendocárdica.

Histologicamente existe expessamento fibroso do endocárdio às custas de colágeno sem fibras elásticas e poucos fibrocitos. Estas camadas fibrosas apresentam em sua superfície pequenas ilhotas de depósitos hialinizados que estão no processo de incorporaçāo. Os músculos papilares sāo cobertos por um expesso endocárdio de colágeno associado a esclerose subendocárdica do miocárdio subjacente. Os aparelhos valvar e subvalvar estāo moderadamente expessados por processo fibro-mixóide.

Os sintomas apresentados são relacionados às disfunçōes ventriculares direita e esquerda.

O início dos sintomas é, na maioria das vezes, recente mas é possível observar períodos de remissāo que são variáveis em duraçāo e que são seguidos por recidivas até o estádio final de insuficiência cardiaca irreversível.

As formas do lado direito sāo caracterizadas por edema periférico, freqüentemente hepatomegalia e ascite importantes.

As formas do lado esquerdo comprometem a pequena circulação e sāo caracterizadas por episódios repetidos de edema dos pulmōes.

A ausculta revela sinais de insuficiência mitral com ou sem insuficiência tricúspide.

O eletrocardiograma não é específico, mas a baixa voltagem dos complexos é notada.

A radiografia de tórax revela a existência de cardiomegalia, a qual é mais intensamente marcada ao nivel atrial.

A cateterização do lado direito do coraçāo mostra igualdade pressórica no ventrículo e no átrio. O traçado tem um "plateau inclinado" o qual é característico da constricçāo. No ventrículo esquerdo, o traçado confirma a disfunção ventricular como uma elevação semelhante ao "plateau" de pressāo diastólica, média e final. Os estudos pós-operatórios mostram curvas de pressōes atrial e ventricular normais, com o desaparecimento do traçado em plano.
A angiocardiografia das cavidades do lado direito revela dilatação atrial com estase do meio de constraste. Existe uma amputaçāo das câmaras de enchimento do ventrículo com passagem direta entre - átrio e o tronco pulmonar. No lado esquerdo o ápex ventricular está deformado assumindo configuraçāo característica que lembra o "coraçāo em ás" e existe associada uma marcada insuficiência mitral com dilatação atrial.

Estudos pós-operatórios demonstraram a opacificação das câmaras de enchimento do ventrículo direito, assim como o retorno ao normal da forma do ventrículo esquerdo.

Em certos terminais a natureza irreversível da moléstia trouxe para discussão a possibilidade da aceitaçāo para o transplante cardíaco.

O tratamento cirúrgico parece oferecer boa chance de sobrevida a longo prazo. No presente estado dos nossos conhecimentos ele pode ser recomendado como método terapêutico de escolha, mesmo nos estágios mais avançados da moléstia.

Baseando-se na análise da casuística e considerando: 1) a elevada mortalidade hospitalar quando se pratica o tratamento cirúrgico convencional da ressecçāo endocardíaca associada a substituiçăo valvar e 2) que os métodos conservadores alternativos (plastias) já iniciados nesta Instituição ${ }^{\circ}$ inicialmente parecem propiciar uma significativa diminuiçāo na mortalidade hospitalr, acompanhada pelo alívio da sintomatologia e melhora da classe funcional, em relação ao pré-operatório, concentramo-nos no objetivo de estabelecer um estudo comparativo entre os dois subgrupos de pacientes: $01^{2}$, de pacientes tratados cirurgicamente com endocardiectomia e substituição da valva atrioventricular e $02^{\circ}$, de pacientes tratados cirurgicamente com endocardiectomia e plastia da valva atrioventricular. A intenção foi de obter subsídios que nos permitissem escolher o método cirúrgico mais eficiente e seguro, possibilitando a melhoria da qualidade de vida e de sobrevida, aos portadores da moléstia.

\section{CASUÍSTICA E MÉTODOS}

Foram analisados, retrospectivamente, 66 pacientes portadores de EMF, tratados cirurgicamente no InCór, no período compreendido entre janeiro de 1978 e dezembro de 1990.

Os pacientes foram incluídos em dois subgrupos: $\left.\mathrm{G}^{\circ}{ }^{\circ}\right)$ os submetidos a ressecçāo endocárdica e substituiçāo valvar e $\mathrm{G}^{2}{ }^{\circ}$ ) os submetidos a ressecçāo endocárdica e plastia valvar, equiparados e comparados quanto a mortalidade hospitalar tardia e curvas atuariais de sobrevida. 
IGLÉZIAS, J. C. R.; DALLAN, L. A.; PEREIRA-BARRETO, A. C.; MADY, C.; LUZ, P. L.; STOLF, N. A. G.; OLIVEIRA, S. A.; PILEGGI, F.; JATENE, A. D. - Tratamento cirúrgico da endomiocárdiofibrose: substituiçāo valvar versus técnicas conservadoras. Rev. Bras. Cir. Cardiovasc., 7(4):269-274, 1992.

A análise estatística foi realizada através do teste de $\mathrm{X}^{2}$ de Pearson e pelo teste de Loog-Rank.

\section{Tratamento Cirúrgico}

Os pacientes foram operados através de toracotomia mediana. A circulaçāo extracorpórea foi estabelecida através da canulaçāo da aorta ascendente e das veias cavas. Foi utilizada a hipotermia moderada obtida por meio da perfusão.

A duraçăo da circulação extracorpórea foi menor que $120^{\prime}$ em $66,66 \%$ e $70,37 \%$ nos pacientes dos grupos 1 e 2, respectivamente. A proteção miocárdica, quando necessária, foi realizada pela infusāo de solução cardioplégica cristalóide, do tipo Saint Thomas, na aorta ascendente. Os procedimentos intracardíacos foram realizados por meio do pinçamento aórtico anóxico intermitente.

A endocardiectomia do ventrículo direito foi realizada após a abertura do átrio direito tomando cuidado no sentido de que fosse preservado o miocárdio subjacente.

Em paciente, mesmo com moléstia biventricular ou com a forma esquerda, a resseçāo endocárdica foi feita pela via atrial, ventricular ou combinada.

O acesso à cavidade ventricular esquerda variou de acordo com o tamanho do átrio esquerdo. Nos pacientes que apresentavam átrio esquerdo dilatado, uma boa exposiçāo foi obtida por atriotomia ampla. Nos demais, o acesso foi feito pela via ventricular ou combinada.

\section{RESULTADOS}

No roteiro para a análise comparativa dos grupos foram incluídos parâmetros clínicos radiológicos, hemodinâmicos, operatórios e de pós-operatório (Tabela 1).

O G1 era formado por 39 pacientes ( 7 masculinos e 32 femininos), apresentava média de idade igual a 37,35 anos ( 9 a 65 ) e em $43,58 \%$ os sintomas haviam se iniciando há mais de 24 meses. Em classe funcional (CF) III e IV pré-operatoriamente estavam $92,30 \%$ deles. Apresentavam a forma univentricular da molestia $15(38,46 \%)$ pacientes e era biventricular nos $24(61,54 \%)$ outros. Além de ressecçāo endocárdica, $12(30,76 \%)$ pacientes se submeteram a dupla substituiçāo valvar, 11 (28,20\%) a substituiçāo tricúspide e $6(15,38 \%)$ se submeteram a substituiçāo valvar e plastia. A mortalidade operatória foi de $(23,07 \%)$ e o tempo médio de seguimento foi igual a 69,34 (33 a 120) meses. No último controle ambulatorial $89,28 \%$ dos pacientes se encontravam em CF I e II.
O G2 era formado por 27 pacientes (11 masculinos e 16 femininos) com média de idade igual a 41,48 anos (5 a 60 ). Em $55,5 \%$ deles os sintomas haviam se iniciado há mais de 24 meses e $100 \%$ se encontravam em CF III e IV pré-operatoriamente. Seis $(22,22 \%)$ pacientes apresentavam a forma univentricular da molestia enquanto em 21 (77,77\%) era biventricular. Além da ressecção endocárdica, $13(48,14 \%)$ pacientes se submeteram a plastia de uma valva, $10(37,03 \%)$ a substituição de duas valvas e $4(14,81 \%)$ foram submetidos somente a ressecçāo endocárdica. A mortalidade hospitalar foi de $(11,11 \%)$ e o tempo médio de seguimento foi de 25 (5-97) meses. No último controle ambulatorial $87,5 \%$ dos pacientes se encontravam em CF III e IV.

\section{COMENTÁRIOS}

Em nosso meio, no InCór, segundo dados publicados por BARRETO et alii ${ }^{1}$ descrevendo a evoluçāo clínica de 108 pacientes portadores de EMF, observaram que no grupo onde se encontravam em CF III e IV a sobrevida foi maior para aqueles que se submeteram ao tratamento cirúrgico. Dentre os fatores associados para a maior mortalidade, destacaram o envolvimento biventricular moderado ou

TABELA 1

PARÂMETROS INCLUIDOS NO ESTUDO COMPARATIVO DA EMF
Sexo
Idade
Dispnéia
4 Edema de membros inferiores
Ascite
Tempo de início dos sintomas
Moléstias associadas
Índice cardiotorácico
Classe funcional pré-operatória

10 Fração de ejeçāo do ventrículo esquerdo (préoperatório)

11 Pressāo diastólica final do ventrículo esquerdo

12 Forma da doença

13 Procedimento operatório (tipo)

14 Duração da circulação extracorpórea

15 Baixo débito cardíaco no pós-operatório

16 Utilizaçāo do cateter de Swan-Ganz

17 Uso de balāo intra-aórtico

18 Assistência ventilatória por período $\geqslant 24$ horas

19 Intercorrências neurológicas no pós-operatório imediato

20 Infecção no pós-operatório

21 Sangramento no pós-operatório que determinou revisāo da hemostasia

22 Situação do paciente após 30 dias

23 Situação clínica do paciente no último controle ambulatorial

24 Classe funcional do paciente no último controle ambulatoria 
IGLÉZIAS, J. C. R.; DALLAN, L. A.; PEREIRA-BARRETO, A. C.; MADY, C.; LUZ, P. L.; STOLF, N. A. G.; OLIVEIRA, S. A.; PILEGGI, F.; JATENE, A. D. - Tratamento cirúrgico da endomiocárdiofibrose: substituição valvar versus técnicas conservadoras. Rev. Bras. Cir. Cardiovasc., 7(4):269-274, 1992.

severo, a fibrose ventricular direita e a presença de insuficiência da valva atrioventricular. Dentre os pacientes tratados cirurgicamente, pela análise da mortalidade hospitalar e a comparaçāo entre as curvas atuariais de sobrevida, identificamos nítida tendência de melhoria para aquele grupo de pacientes tratado pela endocardiectomia associada a plastia das valvas atrioventriculares.

Ainda no InCór, em análise anterior realizada por MADY et alii ${ }^{5}$, em 86 pacientes portadores de EMF e comparando o subgrupo tratado cirurgicamente com o subgrupo tratado clinicamente, observaram que quando os pacientes se encontravam em CF III e IV deveriam ser considerados para a operaçāo, embora a mortalidade hospitalar fosse elevada $(27,3 \%)$. Nesta situação também o benefício sintomático e a melhoria na classe funcional foi bastante evidente.

OLIVEIRA et alii ${ }^{\circ}$ propondo nova abordagem para os pacientes portadores da EMF sugere que a ressecção da fibrose endocárdica pode ser indicada precocemente na evolução clínica da moléstia e deve ser feita com a preservaçāo das valvas atrioventriculares.

MORAES et alii ${ }^{8}$ analisando 30 pacientes portadores de EMF tratados cirurgicamente relatam mortalidade hospitalar de $20 \%$ e concluem que a operação, embora considerada um procedimento paliativo, parece se tratar da terapêutica de escolha devido, nem sempre o tratamento medicamentoso ser efetivo, o prognóstico ser grave, a moléstia afetar somente o coração e o tratamento cirúrgico ser facilmente realizável.

VALICITHAN et alii ${ }^{11}$ analisando 46 pacientes portadoras de EMF referem mortalidade operatória e tardia nos 2 primeiros anos de $21,7 \%$ e $13 \%$, respectivamente.
METRAS et alii ${ }^{6}$ analisando 55 pacientes portadores de EMF tratados cirurgicamente relatam mortalidade hospitalar de $16 \%$ devido também ao baixo débito cardíaco. Coricluem ainda que o tratamento cirúrgico é benéfico, porém a mortalidade operatória é alta e que o procedimento valvar conservador tem se mostrado possível, mesmo naqueles pacientes portadores de insuficiência mitral severa e forma esquerda da moléstia.

METRAS et alii ${ }^{7}$ analisando quatro casos de pacientes africanos, adolescentes submetidos ao tratamento cirúrgico da EMF, também concluem que - tratamento conservador (plastia valvar e encurtamento de cordas) parece ser o método de escolha para o tratamento da insuficiência mitral nas formas localizadas da doença onde a endocardiectomia nāo se torna necessária na regiảo dos músculos papilares.

WOOD et alii ${ }^{12}$ relatando o caso de uma gestante portadora de EMF e insuficiência mitral severa, corroboram nossa impressão, ao relatar que a plastia valvar mitral associada a endocardiectomia resultou no alívio sintomático, em gestaçāo não complicada. Concluem que a reconstrução da valva atrioventricular representa uma boa alternativa à substituiçāo valvar, neste tipo de moléstia.

DUBOST et alii ${ }^{4}$, analisando o resultado do tratamento cirúrgico em 32 pacientes portadores de EMF concluem ser a endocardiectomia o melhor método corrente para o tratamento da moléstia.

Concluímos, portanto, baseando-se em nossa casuística, que o tratamento de eleição para o tratamento cirúrgico da EMF consiste na ressecçāo endocárdica associada às técnicas conservadoras para as valvas atrioventriculares. 
IGLÉZIAS, J. C. R.; DALLAN, L. A.; PEREIRA-BARRETO, A. C.; MADY, C.; LUZ, P. L.; STOLF, N. A. G.; OLIVEIRA, S. A.; PILEGGI, F.; JATENE, A. D. - Tratamento cirúrgico da endomiocárdiofibrose: substituiçāo valvar versus técnicas conservadoras. Rev. Bras. Cir. Cardiovasc., 7(4):269-274, 1992.

\section{RBCCV 44205-189}

IGLÉzIAS, J. C. R.; DALLAN, L. A.; PEREIRA-BARRETO, A. C.; MADY, C.; LUZ, P. L.; STOLF, N. A. G.; OLIVEIRA, S. A.; PILEGGI, F.; JATENE, A. D. - Endomyocardial fibrosis surgical treatment: substitution versus conservative techniques. Rev. Bras. Cir. Cardiovasc., 7(4):269-274, 1992.

ABSTRACT: Sixty-six (66 patients) with endomyocardial fibrosis underwent surgical treatment at the Heart Institute between January 1978 and June 1990. A study involving two subgroups was designed with the aim to compare the operative techniques; 1) patients submitted to endocardiectomy and valvar substitution; and 2) patients submitted to endocardiectomy and valvoplasty. Clinical, radiologic, hemodynamic, surgical and postoperative parameters were included in the protocol. It is a retrospective study, an statistical analysis was performed using Pearson's $X^{2}$ test and Loog-Rank test. At this time, the comparative analysis does not reveal significant statistical differences between the two subgroups, both regarding nosocomial mortality and late survival, however comparison of the actuarial survival curves allows to identify a tendency towards improvement, with decrease in the nosocomial mortality and a great late survival, when the conservative technique for surgical treatment of endomocardial fibrosis is used. Therefore, endocardiectomy associated to atrioventricular valvoplasty for surgical treatment of the patients with endomyocardial fibrosis is recommended.

DESCRIPTORS: endomyocardial fibrosis, surgery; heart valves, surgery.

\section{REFERÊNCIAS BIBLIOGRÁFICAS}

BARRETO, A. C. P.; LUZ, P. L.; OLIVEIRA, S. A.; STOLF, N. A. G.; MADY, C.; BELLOTTI, G.; JATENE, A. D.; PILEGGI, F. - Determinants of survival in endomyocardial fibrosis. Circulation, 80 (Supl. 1): 177-181, 1989.

2 DUBOST, C.; MAURICE, P.; GERBAUX, A.; BERTRAND, E.; RULLIERE, R.; VIAL, F.; BARRILON, A.; PRIGENT, C.; CARPENTIER, A.; SOYER, R. The surgical treatment of constrictive fibrosous endocarditis. Ann. Surg., 184: 303-307, 1976.

3 DUBOST, C.; MAURICE, P.; GERBAUX, A.; RULLIERE, R.; BARILLON, A.; BERTRAND, E.; VIAL, F.; PRIGENT, C.; CARPENTIER, A.; SOYER, R. L. Endocardite fibruse constrictive: traitement chirurgicale. Arch. Mal. Coeur, 70: 155-162, 1977.

4 DUBOST, C.; PRIGENT, C.; GERBAUX, A.; MAURICE, P.; PASSELECQ, J.; RULLIER, R.; CARPENTIER, A.; BLOCHE, A. - Surgical treatment of constrictive fibrosis endocarditis. J. Thorac. Cardiovasc. Surg., 82: $585-591,1981$.

5 MADY, C.; PEREIRA BARRETO, A. C.; ARTEGA FERNANDES, E.; BELLOTTI, G.; JATENE, A. D.; PILEGGI, F. - Endomyocardial fibrosis: outcome according to the rend of treatment Eur. Heart J., o (Supl. 1): 296, 1988. (Resumo).

METRAS, D.; QUATARA, K.; COULIBALY, A. D.; TOU$Z E$, J. E. - Left endomyocardial fibrosis with severe mitral insuficiency: the case for mitral valve repair: a report of 4 cases. Thorac. Cardiovasc. Surg., 31: 297-301, 1983.

7 METRAS, N.; COULIBALY, A. N.; QUATTARA, K. - The surgical treatment of endomyocardial fibrosis: results in 55 patients. Circulation, 72 (Supl. 2): 274-279, 1285.

8 MORAES, C. R.; BUFFOLO, E.; LIMA, R.; VICTOR, E.; LIMA, V.; ESCOBAR, M.; RODRIGUES, J.; SARAIVA, L.; ANDRADE, J. C. - Surgical treatment of endomyocardial fibrosis. J. Thorac. Cardiovasc. Surg., 85: 738-745, 1983.

9 OLIVEIRA, S. A.; BARRETO, A. C.; MADY, C.; DALLAN, L. A.; LUZ, P. L.; JATENE, A. D.; PILEGGI, F. Endomyocardial fibrosis: a new surgical aproach. $J$. Am. Coll. Cardiol., 16: 1246, 1990.

10 PRIGENT, C. L.; BACULARD, P.; CARPENTIER, A.; PASSELECO, J.; DUBOST, C. H. - Endocardite fibrosis du ventricule gauche chez un filarier: cure chirurgicale. Arch. Mal. Coeur, 66: 101-105, 1973.

11 VALICITHAN, M. S.; BALAKRISHMAN, K. G.; SANKARKUMAR, R.; KARTH, C. C. - Surgical treatment of endomyocardial fibrosis. Ann. Thorac. Surg., 43: 68-73, 1987.

12 WOOD, A. E.; BOYLE, D.; O'HARA, M. D.; CLELAND, J. - Mitral annuloplasty in endomyocardial fibrosis: alternative to valve replacement. Ann. Thorac. Surg., 34: 446-451, 1982.

\section{Discussāo}

PROF. CARLOS MORAES

Recife, PE

Eu queria, inicialmente, cumprimentar o Dr. Iglezias e colaboradores pelo trabalho apresentado. Concordo inteiramente com a conclusão dos autores de que, no tratamento cirúrgico da endomio- 

F.; JATENE, A. D. - Tratamento cirúrgico da endomiocárdiofibrose: substituiçảo valvar versus técnicas conservadoras. Rev. Bras. Cir. Cardiovasc., 7(4):269-274, 1992.

cardiofibrose (EMF), a plastia valvar associada à endocardiectomia determina melhores resultados imediatos e tardios. Contudo, penso que, na análise da cirurgia da EMF, outras variáveis devem ser levadas em consideraçāo, uma vez que o espectro da doença é muito amplo. A situação nutricional dos pacientes no pré-operatório e, muito especialmente, a localização da doença são apenas alguns dos fatores que influenciam no resultado cirúrgico. Quando analisamos nosso material, costumamos sempre dividi-lo em 3 grupos: o grupo com doença biventricular, o grupo com doença isolada do ventrículo direito e o grupo com doença apenas ventricular esquerda. Isto porque o resultado cirúrgico é completamente diferente. Em nossa experiência, a mortalidade foi de $29 \%$ nos pacientes com doença biventricular, de $12,7 \%$ nos pacientes com lesāo do lado direito e de apenas $6,2 \%$ nos casos com lesāo isolada do ventrículo esquerdo. Essa diferença foi estatisticamente significativa. Gostaria de perguntar ao Dr. Iglézias se, na análise dos seus resultados, observou esse mesmo tipo de diferença e se outras variáveis, exceto a técnica cirúrgica, foram estudadas. Mais uma vez, parabenizo os autores pelo excelente trabalho apresentado.

\section{DR. IGLÉZIAS}

\section{(Encerrando)}

Agradeço a gentileza do Prof. Carlos Moraes, pela valiosa contribuiçāo, baseada em sua casuística. O objetivo do nosso projeto foi o de comparar as duas técnicas operatórias, usando o método de eleição, ou seja, aquela que nos proporcionasse menor mortalidade hospitalar e melhora na situaçăo funcional a longo prazo. Baseados nisto, dividimos os 66 pacientes da casuística em subgrupos: G1 - pacientes submetidos a endocardiectomia e substituiçāo (ōes) valvar (es) = 39 pacientes; e G2 - dos pacientes submetidos a endocardiectomia e plastia (s) valvar (es) $=27$ pacientes. Nossos resultados apontaram para a técnica de plastia, associada à endocardiectomia como o método de eleição e esta vem se confirmando, à medida em que a casuística aumenta. Considerando a divisāo feita pelo Prof. Carlos Moraes, em três subgrupos: 1) pacientes portadores da moléstia em sua forma biventricular; 2) pacientes com acometimento isolado do ventrículo direito e, 3) pacientes com acometimento isolado do ventrículo esquerdo, com resultados operatórios diferentes, podemos afirmar que, em nossa casuística, houve um predomínio da forma biventricular nos dois subgrupos: $24(61,54 \%)$ e $21(77,77 \%)$, respectivamente. Embora nenhum tratamento estatístico específico tenha sido realizado para comparar os resultados obtidos com os pacientes portadores da moléstia em suas formas uni ou biventricular em nossa casuística, minha impressāo pessoal é de que o resultado nas formas univentriculares seja melhor do que aquele obtido para os portadores da moléstia em sua forma biventricular. Reiteradamente, agradeço a apreciação feita pelo Prof. Carlos Moraes e observo que nenhuma outra análise foi feita, que nāo aquela voltada para a comparaçāo entre as duas técnicas operatórias. 